Proceedings of the ASME 2019

International Design Engineering Technical Conferences

and Computers and Information in Engineering Conference

IDETC/CIE2019

August 18-21, 2019, Anaheim, CA, USA

\title{
IDETC2019-97541
}

\section{SECOND ORDER RELIABILITY METHOD FOR TIME-DEPENDENT RELIABILITY ANALYSIS USING SEQUENTIAL EFFICIENT GLOBAL OPTIMIZATION}

\author{
Zhangli Hu \\ Department of Mechanical and Aerospace Engineering \\ Missouri University of Science and Technology \\ Rolla, MO, USA
}

\author{
Xiaoping $\mathrm{Du}^{1}$ \\ Department of Mechanical and Energy Engineering \\ Indiana University - Purdue University Indianapolis \\ Indianapolis, IN, USA
}

\begin{abstract}
Reliability depends on time if the associated limit-state function includes time. A time-dependent reliability problem can be converted into a time-independent reliability problem by using the extreme value of the limit-state function. Then the first order reliability method can be used but it may produce a large error since the extreme limit-state function is usually highly nonlinear. This study proposes a new reliability method so that the second order reliability method can be applied to timedependent reliability analysis for higher accuracy while maintaining high efficiency. The method employs sequential efficient global optimization to transform the time-dependent reliability analysis into the time-independent problem. The Hessian approximation and envelope theorem are used to obtain the second order information of the extreme limit-state function. Then the second order saddlepoint approximation is use to evaluate the reliability. The accuracy and efficiency of the proposed method are verified through numerical examples.
\end{abstract}

\section{INTRODUCTION}

Reliability is the probability that a product performs its intended function under specified conditions over a period of time [1]. Higher reliability means a lower chance of failure. It is especially critical to maintain high reliability because failures may be costly and catastrophic. Predicting reliability during a design stage is therefore imperative for many products.

For many engineering applications, reliability depends on time if the associated limit-state function involves timedependent parameters, such as time-variant loads and the deterioration of material properties. For example, the wave loads on offshore structures are time-dependent since the typical wave heights and periods change randomly over time [2]; the material and dimensional properties of concrete structures vary with respect to time due to the time-dependent chloride corrosion damage $[3,4]$; for mechanisms, the motion error involves timedependent input motion $[5,6]$.

Extensive research has been conducted on time-dependent reliability analysis. Existing time-dependent reliability methodologies can be roughly classified into three group. The first group is Rice's formula based methods, whose key step is the computation of the upcrossing rate. For instance, a PHI2 method was developed to compute the time-variant reliability [7]. Hu and Du proposed a time-dependent reliability method for hydrokinetic turbine blades [8]. Besides, many other empirical modifications [5, 9-14] have also been made. This group has advantages over other groups for its efficiency. But it may produce large errors when upcrossings are strongly dependent.

The second group includes simulation-based methods using surrogate models. Most of these methods build a surrogate model to replace the original limit-state function by evaluating the response variable at a number of points predefined through Design of Experiment (DoE) [15-17]. Then Monte Carlo simulation (MCS) is performed based on the surrogate model. The methods include artificial neural networks (ANN) [18, 19], polynomial chaos expansions (PCE) [20, 21], and Gaussian process based method, also known as Kriging model based methods [22-26]. This group can evaluate the time-dependent reliability accurately if the surrogate model is well trained. Nevertheless, this may result in a high computational cost.

${ }^{1} 723$ W. Michigan Street, SL 260G, Indianapolis, IN 46202, U.S.A., tel: 1-317-278-3113, e-mail: duxi@iu.edu

This is the author's manuscript of the article published in final edited form as:

Hu, Z., \& Du, X. (2019, November 25). Second Order Reliability Method for Time-Dependent Reliability Analysis Using Sequential Efficient Global Optimization. ASME 2019 International Design Engineering Technical Conferences and Computers and Information in Engineering Conference. 
The third group contains the methods that convert timedependent reliability analysis into the time-independent reliability analysis using the extreme value of the timedependent limit-state function. If the distribution of the extreme value can be estimated accurately, the accuracy of this group is higher than the first group. The typical methods in this group are extreme value response method [22, 27], extreme value distribution method [28], composite limit-state function method [29], and the envelope function method [6]. However, it is often a challenging task to obtain the distribution of the extreme value accurately and efficiently.

We propose a new time-dependent method using sequential efficient global optimization (SEGO). The new method first converts the time-dependent problem into a time-independent counterpart by using the extreme value. Then the Hessian approximation and envelope theorem are employed to obtain the second order approximation to the extreme value. Finally the second order saddlepoint approximation (SOSPA) [30, 31] is used to estimate the distribution of the extreme value. The new method is termed as SEGO/SOSPA. It improves the accuracy by using second order approximation to the extreme value of the limit-state function and maintains high efficiency by using SEGO.

The reminder of this paper is organized as follows: Section 2 reviews the theoretical background of this work. Then the new SEGO/SOSPA method is discussed in Section 3 followed by three examples in Section 4. Conclusions are given in Section 5.

\section{REVIEW OF FUNDAMENTAL METHODOLOGIES}

In this section, we briefly review the basic definition of time dependent reliability. We also discuss the commonly used first order reliability method (FORM).

\subsection{Time-Dependent Reliability}

In this work, we consider a limit-state function given by

$$
Y=g(\mathbf{X}, t)
$$

where $g(\mathbf{X}, t)$ is explicit with respect to time $t$, $\mathbf{X}=\left[X_{1}, \ldots, X_{N}\right]^{T}$ is an $\mathrm{N}$-dimensional vector of independent random variables.

For a given period of time $[0, T]$, the reliability is defined by

$$
R(0, T)=\operatorname{Pr}\{g(\mathbf{X}, t) \geq 0, \forall t \in[0, T]\}
$$

in which $\forall$ means "for all".

The associated probability of failure is given by

$$
p_{f}(0, T)=\operatorname{Pr}\{g(\mathbf{X}, t)<0, \exists t \in[0, T]\}
$$

where $\exists$ means "there exists at least one".

\subsection{First Order Reliability Method (FORM)}

FORM is the most commonly used method in timedependent reliability analysis since it can convert the general non-Gaussian process into an equivalent Gaussian process [32].

$\mathbf{X}$ is transformed into standard normal variables $\mathbf{U}$. Then the most probable point (MPP) $\mathbf{u}_{M P P}$ is identified by the following model:

$$
\begin{cases}\min & \sqrt{\mathbf{U}^{T}} \\ \text { s.t. } & g(\mathbf{X}, t)=g(T(\mathbf{U}), t)=0\end{cases}
$$

in which $T(\cdot)$ is an operator of the transformation from $\mathbf{U}$ to $\mathbf{X}$.

The limit-state function is linearized at $\mathbf{u}_{M P P}$ by

$$
\begin{aligned}
g(T(\mathbf{U}), t) & =g\left(\mathbf{u}_{M P P}, t\right)+\left.\sum_{i=1}^{N} \frac{\partial g}{\partial U_{i}}\right|_{\mathbf{U}=\mathbf{u}_{M P P}}\left(U_{i}-u_{M P P, i}\right) \\
& =\nabla g\left(\mathbf{u}_{M P P}, t\right)\left(\mathbf{U}-\mathbf{u}_{M P P}\right)
\end{aligned}
$$

where $\nabla g\left(\mathbf{u}_{M P P}, t\right)=\left[\left.\frac{\partial g}{\partial U_{1}}\right|_{\mathbf{U}=\mathbf{u}_{M P P}}, \ldots,\left.\frac{\partial g}{\partial U_{N}}\right|_{\mathbf{U}=\mathbf{u}_{M P P}}\right]^{T}$ is the gradient vector, and $u_{M P P, i}$ is the $i$-th component of $\mathbf{u}_{M P P}$.

Finally, the probability of failure can be estimated by

$$
\begin{aligned}
p_{f} & =\operatorname{Pr}\{g(\mathbf{X}, t)<0, \exists t \in[0, T]\} \\
& =\operatorname{Pr}\left\{g\left(\mathbf{u}_{M P P}, t\right)+\left.\sum_{i=1}^{N} \frac{\partial g}{\partial U_{i}}\right|_{\mathbf{U}=\mathbf{u}_{M P P}}\left(U_{i}-u_{i M P P}\right)<0\right\} \\
& =\operatorname{Pr}\{\beta(t)+\boldsymbol{\alpha}(t) \mathbf{U}<0, \exists t \in[0, T]\}
\end{aligned}
$$

in which $\beta(t)$ is the time-dependent reliability index

$$
\beta(t)=\left\|\mathbf{u}_{M P P}\right\|
$$

And $\boldsymbol{\alpha}(t)$ is the time-dependent unit gradient vector

$$
\boldsymbol{\alpha}(t)=\frac{\nabla g\left(\mathbf{u}_{M P P}, t\right)}{\left\|\nabla g\left(\mathbf{u}_{M P P}, t\right)\right\|}=\left[\alpha_{1}(t), \alpha_{2}(t), \ldots, \alpha_{N}(t)\right]^{T}
$$

As Eq. (6) shows, the non-Gaussian process $g(\mathbf{X}, t)$ has been transformed into an equivalent Gaussian process represented as a sum of standard normal random variables. A common method is to build the surrogate models of $\beta(t)$ and $\alpha(t)$ with respect to $t$ and then to use MCS to estimate the probability of failure. However, it might be computational expensive to build accurate surrogate models.

\section{SEGO/SOSPA}

The objective of this study is to improve the accuracy of the time-dependent reliability analysis by employing the secondorder approximation. The central idea is to convert the timedependent problem into a time-independent problem using sequential efficient global optimization (SEGO). The second order approximation is obtained by using the Hessian approximation and envelope theorem. Then the timeindependent problem is addressed with the second-order saddlepoint approximation (SOSPA) [30].

\subsection{Overview}

The time-dependent probability of failure can be evaluated through the extreme value of the limit-state function, expressed by [27]

$$
\begin{aligned}
p_{f}(0, T) & =\operatorname{Pr}\{g(\mathbf{X}, t)<0, \exists t \in[0, T]\} \\
& =\operatorname{Pr}\left\{\min _{t \in[0, T]} g(\mathbf{X}, t)<0\right\}
\end{aligned}
$$


The extreme limit-state function, also known as the envelope function [6], or the composite limit-state function [29], $\min _{t \in[0, T]} g(\mathbf{X}, t)$ is obtained by

$$
G(\mathbf{X})=\min _{t \in[0, T]} g(\mathbf{X}, t)=g(\mathbf{X}, \tilde{t}(\mathbf{X}))
$$

where $G(\mathbf{X})$ is the global minimal value of $g(\mathbf{X}, t)$ with respect to $t . G(\mathbf{X})$ is time independent and only depends on $\mathbf{X} . \tilde{t}$ is the time instant when the global minimal value occurs. $\tilde{t}$ is a function of $\mathbf{X}$.

$$
\tilde{t}=\left\{t \mid \min _{t \in[0, T]} g(\mathbf{X}, t)\right\}
$$

Now Eq. (9) can be converted into the following timeindependent problem:

$$
\begin{aligned}
p_{f}(0, T) & =\operatorname{Pr}\{g(\mathbf{X}, t)<0, \exists t \in[0, T]\} \\
& =\operatorname{Pr}\{G(\mathbf{X})<0\}
\end{aligned}
$$

Then a time-independent reliability method can be applied after the conversion. In this work, we assume that $G(\mathbf{X})$ exists and is continuously differentiable.

\subsection{SEGO}

In this subsection, we discuss the proposed method.

\subsubsection{Sequential Optimization}

It is very difficult to analytically obtain the extreme limitstate function $G(\mathbf{X})$. So FORM is generally used to approximate $G(\mathbf{X})$, and the MPP of $G(\mathbf{X})$ is found by using the following optimization model:

$$
\begin{cases}\min & \sqrt{\mathbf{U} \mathbf{U}^{T}} \\ \text { s.t. } & G(T(\mathbf{U}))=\min _{t \in[0, T]} g(T(\mathbf{U}), t)=0\end{cases}
$$

Eq. (13) is formulated as a double loop structure. The inner loop is the global optimization with respect to time $t$, while the outer loop is the MPP search with respect to $\mathbf{U}$. The computational cost of the double loop optimization is very high.

Inspired by sequential optimization and reliability assessment (SORA) [33], we use a sequential strategy to decouple the global optimization from the MPP search and perform the two loops sequentially. In the first cycle, FORM is used to locate the MPP $\mathbf{u}_{M P P}^{(1)}$ at the initial time $t_{0}$.

$$
\begin{cases}\min & \sqrt{\mathbf{U U}^{T}} \\ \text { s.t. } & g\left(T(\mathbf{U}), t_{0}\right)=0\end{cases}
$$

After the MPP $\mathbf{u}_{M P P}^{(1)}$ is obtained, the global optimization is performed by fixing $\mathbf{U}$ at the $\mathbf{u}_{M P P}^{(1)}$, and optimal time is obtained as $\tilde{t}^{(1)}$.

$$
\tilde{t}^{(1)}=\underset{t \in[0, T]}{\arg \min } g\left(T\left(\mathbf{u}_{M P P}^{(1)}\right), t\right)
$$

In the second cycle, the new MPP $\mathbf{u}_{M P P}^{(2)}$ is located at the time instant $\tilde{t}^{(1)}$ using Eq. (14). And then the extreme value time is updated to $\tilde{t}^{(2)}$ by performing global optimization at $\mathbf{u}_{M P P}^{(2)}$.

$$
\tilde{t}^{(2)}=\underset{t \in[0, T]}{\arg \min } g\left(T\left(\mathbf{u}_{M P P}^{(2)}\right), t\right)
$$

This process is repeated until convergence. The global optimization is discussed in Sec. 3.2.2.

The flowchart of the above procedure is illustrated in Fig. 1.

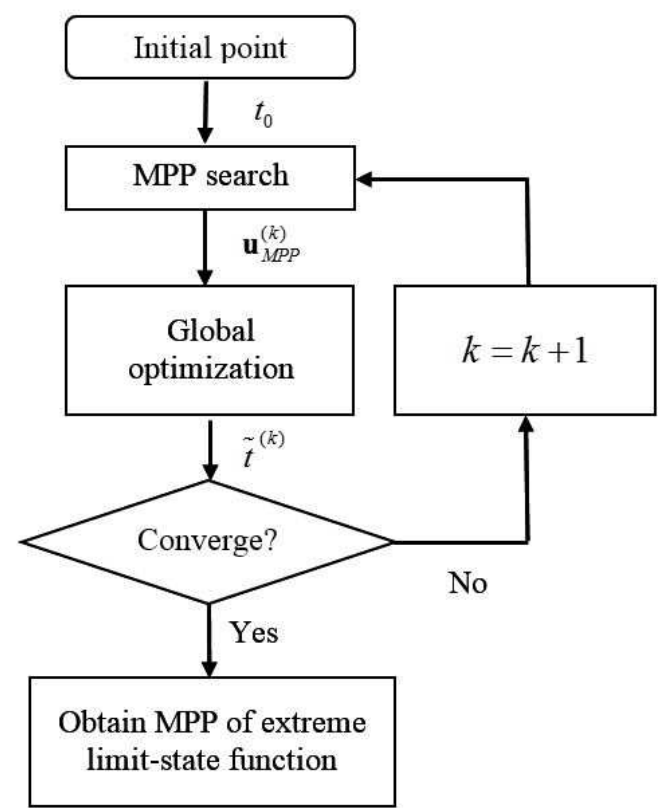

Fig. 1 Flowchart of sequential optimization

\subsubsection{Efficient Global Optimization (EGO)}

The global optimization method used in this study is the efficient global optimization (EGO) [22, 27, 34]. EGO has been widely used in various areas $[35,36]$ because it can search for the global optimum with high computational efficiency. In this work, we search for a time instant where $Y=g\left(T\left(\mathbf{u}_{M P P}\right), t\right)$ is minimized. Recall that $\mathbf{u}_{M P P}$ is fixed during the optimization process, and then $g\left(T\left(\mathbf{u}_{M P P}\right), t\right)$ is a one-dimensional function. We denote this function as $g(t)$; namely, $g(t)=g\left(T\left(\mathbf{u}_{M P P}\right), t\right)$. We then create its surrogate model $\hat{g}(t)$ as follows:

$$
\hat{y}=\hat{g}(t)=\hat{g}\left(T\left(\mathbf{u}_{M P P}\right), t\right)=F(t)^{T} \gamma+Z(t)
$$

where $F(t)^{T} \gamma$ is a deterministic term, $F(t)$ is a vector of regression functions, $\gamma$ is a vector of regression coefficients, and $Z(t)$ is a stationary Gaussian process with zero mean and a covariance given by

$$
\operatorname{Cov}\left[Z\left(t_{1}\right), Z\left(t_{2}\right)\right]=\sigma_{Z}^{2} R\left(t_{1}, t_{2}\right)
$$

in which $\sigma_{Z}^{2}$ is process variance, and $R(\cdot, \cdot)$ is the correlation function. 
The output of the surrogate model is a Gaussian random variable following

$$
\hat{y}=\hat{g}(t) \sim N\left(\mu(t), \sigma^{2}(t)\right)
$$

where $\mu(t)$ and $\sigma^{2}(t)$ are the mean and standard deviation of $\hat{y}$. If $t$ is a training point, $\mu(t)=g(t)$ and $\sigma(t)=0$. This means that the surrogate model is exact at a point where the model is trained.

After building the initial model, the expected improvement (EI) metric is used to identify the new training point with the highest probability to produce a better extreme value of the response. The improvement is defined by

$$
I=\max \left(y^{*}-y, 0\right)
$$

in which $y^{*}=\min _{i=1,2, \ldots, k} g\left(t_{i}\right)$ is the current minimum response obtained from the sampled training points.

Thus its expectation or EI is computed by [34]

$$
\begin{aligned}
\mathrm{EI}(t) & =E\left[\max \left(y^{*}-y, 0\right)\right] \\
& =\left(y^{*}-\mu(t)\right) \Phi\left(\frac{y^{*}-\mu(t)}{\sigma(t)}\right)+\sigma(t) \phi\left(\frac{y^{*}-\mu(t)}{\sigma(t)}\right)
\end{aligned}
$$

where $\Phi(\cdot)$ and $\phi(\cdot)$ are the cumulative distribution function

\begin{tabular}{|c|c|}
\hline Steps & Procedure \\
\hline 1 & $\begin{array}{l}\text { Generate initial training points } \mathbf{t}^{s}=\left[t_{1}, t_{2}, \ldots, t_{k}\right] \text { and } \\
\text { compute the response of limit-state function } \\
\mathbf{y}^{s}=\left[g\left(t_{1}\right), g\left(t_{2}\right), \ldots, g\left(t_{k}\right)\right]\end{array}$ \\
\hline 2 & $\begin{array}{l}\text { Construct a surrogate model } \hat{y}=\hat{g}(t) \text { using } \\
\left\{\mathbf{t}^{s}, \mathbf{y}^{s}\right\}\end{array}$ \\
\hline 3 & $\begin{array}{l}\text { Find the global minimum } y^{*}=\min _{i=1,2, \ldots, k} g\left(t_{i}\right) \text { and } \\
\text { corresponding time } t^{*}\end{array}$ \\
\hline 4 & $\begin{array}{l}\text { Search for } t_{k+1}=\arg \max _{t} \operatorname{EI}(t) \text {, where } \operatorname{EI}(t) \text { is } \\
\text { computed by Eq. (21) }\end{array}$ \\
\hline 5 & $\begin{array}{l}\text { Compare } \max _{t} \operatorname{EI}(t) \text { with } \varepsilon_{\mathrm{EI}}: \text { if } \max _{t} \mathrm{EI}(t)<\varepsilon_{\mathrm{EI}} \\
\text { stop and give the final optimum } y^{*} \text { and } t^{*} \\
\text { otherwise, go to next step }\end{array}$ \\
\hline 6 & $\begin{array}{l}\text { Update } \mathbf{y}^{s}=\left[\mathbf{y}^{s}, g\left(t_{k+1}\right)\right] \text { and } \mathbf{t}^{s}=\left[\mathbf{t}^{s}, t_{k+1}\right] \text {, and } \\
\text { repeat steps 2-5 }\end{array}$ \\
\hline
\end{tabular}
(CDF) and probability density function (PDF) of a standard normal variable, respectively.

Table 1 Algorithms of EGO

The new training point $t_{k+1}$ is identified as the time that maximizes the expected improvement.

$$
t_{k+1}=\arg \max _{t} \mathrm{EI}(t)
$$

The procedure of EGO is described in Table 1. More details can be found in Ref. [34].
In this work, the convergence criterion of EGO $\varepsilon_{\mathrm{EI}}$ is chosen as $\varepsilon_{\mathrm{EI}}=\left|y^{*}\right| \times 2 \%$.

By combining sequential strategy with EGO, the MPP $\mathbf{u}^{*}$ of extreme limit-state function $G(\mathbf{X})$ can be obtained efficiently by solving Eq. (13). If FORM is used, the probability of failure is estimated by

$$
\begin{aligned}
p_{f}(0, T) & =\operatorname{Pr}\{g(\mathbf{X}, t)<0, \exists t \in[0, T]\} \\
& =\operatorname{Pr}\{G(\mathbf{X})<0\} \\
& =\Phi\left(-\beta_{F}^{*}\right)
\end{aligned}
$$

where $\beta_{F}^{*}=\left\|\mathbf{u}^{*}\right\|$ is the first order reliability index of the extreme limit-state function.

Since the above method uses FORM and SEGO, we denote this method as SEGO/FORM. In general, the extreme limit-state function can be highly nonlinear and SEGO/FORM may not be accurate enough. In Sec. 3.3, we discuss how to develop a second-order approximation method. This method uses the Hessian approximation and envelope theorem to obtain the second order information of the extreme limit-state function. Then SOSPA is used to estimate the probability of failure.

\subsection{Hessian Approximation and Envelope Theorem}

The second-order approximation requires the Hessian matrix. But it is challenging to calculate the Hessian because it consists of second derivatives of the extreme limit-state function with respect to random input variables $\mathbf{X}$. Hence a quasiNewton approach $[37,38]$ is introduced in this work to approximate the Hessian matrix. This Hessian approximation method can take advantage of the MPP search information in SEGO, leading to high efficiency. [38]

The Hessian matrix is updated using the following formulas

$$
\mathbf{H}^{(k+1)}=\mathbf{H}^{(k)}+\frac{\left(\mathbf{r}^{(k)}-\mathbf{H}^{(k)} \mathbf{s}^{(k)}\right)\left(\mathbf{r}^{(k)}-\mathbf{H}^{(k)} \mathbf{s}^{(k)}\right)^{T}}{\left(\mathbf{r}^{(k)}-\mathbf{H}^{(k)} \mathbf{s}^{(k)}\right)^{T} \mathbf{s}^{(k)}}
$$

where

$$
\left\{\begin{array}{l}
\mathbf{s}^{(k)}=\mathbf{u}_{M P P}^{(k+1)}-\mathbf{u}_{M P P}^{(k)} \\
\mathbf{r}^{(k)}=\nabla G^{(k+1)}-\nabla G^{(k)}
\end{array}\right.
$$

in which $\mathbf{u}_{M P P}^{(k)}$ represents the MPP at current iteration $k$ used in the SEGO, $\nabla G^{(k)}=\left[\partial G^{(k)} / \partial U_{1}, \ldots, \partial G^{(k)} / \partial U_{n}\right]^{T}$ is the gradient vector of the extreme limit-state function, and $\mathbf{s}^{(k)}$ and $\mathbf{r}^{(k)}$ are the variation of the MPP and the gradient between two successive iterations, respectively.

The approximated Hessian is expected to converge to the true Hessian as the MPP reaches the true MPP. However, SEGO does not provide the gradient information of the extreme limitstate function, and extra computational effort is needed. In this case, the finite difference method is used. 


$$
\begin{aligned}
\frac{\partial G}{\partial U_{i}} & =\frac{G\left(u_{i}+\Delta u_{i}\right)-G\left(u_{i}\right)}{\Delta u_{i}} \\
& =\frac{\min _{t} g\left(u_{i}+\Delta u_{i}, t\right)-\min _{t} g\left(u_{i}, t\right)}{\Delta u_{i}}
\end{aligned}
$$

As Eq. (26) indicates, $\min _{t} g\left(u_{i}+\Delta u_{i}, t\right)$ needs additional global optimization at $u_{i}+\Delta u_{i}$. Directly using the finite difference method will increase $N$ times of global optimization at each iteration in order to obtain the gradient. This is very computationally expensive.

To reduce the computational cost, we use the envelope theorem, which is a widely used method in economic optimization field $[39,40]$. The envelope theorem can connect the derivative of extreme limit-state function with the derivative of original limit-state function.

$$
\frac{\partial}{\partial U_{i}} G(\mathbf{U})=\frac{\partial}{\partial U_{i}} g(\mathbf{U}, \tilde{t}(\mathbf{U}))=\left.\frac{\partial}{\partial U_{i}} g(\mathbf{U}, t)\right|_{t=\tilde{t}(\mathbf{U})}
$$

Eq. (27) indicates that the gradient of the extreme limit-state function (the envelope function) at $\mathbf{U}$ equals to the gradient of the original limit-state function at time instant $t=\tilde{t}(\mathbf{U})$.

Eq. (26) becomes

$$
\begin{aligned}
\frac{\partial G}{\partial U_{i}} & =\frac{G\left(u_{i}+\Delta u_{i}\right)-G\left(u_{i}\right)}{\Delta u_{i}} \\
& =\left.\frac{g\left(u_{i}+\Delta u_{i}, t\right)-g\left(u_{i}, t\right)}{\Delta u_{i}}\right|_{t=\tilde{t}(\mathbf{u})}
\end{aligned}
$$

Then only $N$ function calls are required in each iteration by using the envelope theorem. This makes the method more efficient.

Combining Eqs. (24) and (28) yields the gradient $\nabla G$ and Hessian matrix $\mathbf{H}$ of the extreme limit-state function. Then the second order reliability method can be used.

\subsection{SOSPA}

Once the MPP $\mathbf{u}^{*}$, gradient $\nabla G$, and Hessian matrix $\mathbf{H}$ of the extreme limit-state function are available, the second approximation to the extreme limit-state function is formulated as

$$
G(\mathbf{U})=a+\mathbf{b}^{T} \mathbf{U}+\mathbf{U}^{T} \mathbf{C} \mathbf{U}
$$

where

$$
\left\{\begin{array}{l}
a=\frac{1}{2}\left(\mathbf{u}^{*}\right)^{T} \mathbf{H} \mathbf{u}^{*}-\nabla G\left(\mathbf{u}^{*}\right)^{T} \mathbf{u}^{*} \\
\mathbf{b}=\nabla G\left(\mathbf{u}^{*}\right)-\mathbf{H} \mathbf{u}^{*} \\
\mathbf{C}=\frac{1}{2} \mathbf{H}
\end{array}\right.
$$

Then SOSPA [30] is employed to estimate the probability of failure, and it is considered in general more accurate than the traditional SORM methods such as Breitung's [41] and Tvedt's methods [42].
After the extreme limit-state function is approximated in Eq. (29), we can obtain the cumulant generating function (CGF).

$$
K(t)=-\beta_{F}^{*} t+\frac{1}{2} t^{2}-\frac{1}{2} \sum_{i}^{n-1} \log \left(1-2 t k_{i}\right)
$$

The derivatives of CGF are

$$
\begin{aligned}
& K^{\prime}(t)=-\beta_{F}^{*}+t+\sum_{i=1}^{n-1} \frac{k_{i}}{1-2 t k_{i}} \\
& K^{\prime \prime}(t)=1+\sum_{i=1}^{n-1} \frac{k_{i}^{2}}{\left(1-2 t k_{i}\right)^{2}}
\end{aligned}
$$

The saddlepoint $t_{s}$ is obtained by solving the following equation:

$$
K^{\prime}(t)=-\beta_{F}^{*}+t+\sum_{i=1}^{n-1} \frac{k_{i}}{1-2 t k_{i}}=0
$$

Then the probability of failure is evaluated by

$$
\begin{aligned}
p_{f}(0, T) & =\operatorname{Pr}\{g(\mathbf{X}, t)<0, \exists t \in[0, T]\} \\
& =\operatorname{Pr}\{G(\mathbf{X})<0\} \\
& =\Phi(w)+\phi(w)\left(\frac{1}{w}-\frac{1}{v}\right)
\end{aligned}
$$

where

$$
\begin{gathered}
w=\operatorname{sgn}\left(t_{s}\right)\left\{2\left[-K\left(t_{s}\right)\right]\right\}^{1 / 2} \\
v=t_{s}\left[K^{\prime \prime}\left(t_{s}\right)\right]^{1 / 2}
\end{gathered}
$$

in which $\operatorname{sgn}\left(t_{s}\right)=+1,-1$ or 0 , depending on whether $t_{s}$ is positive, negative, or zero.

Saddlepoint approximation has several excellent features. It yields an extremely accurate probability estimation, especially in the tail area of a distribution [43-46]. More details can be found in Ref. [30].

\subsection{SEGO/SOSPA Procedure}

The detailed steps of SEGO/SOSPA are summarized below.

Step 1: Set $k=1$. Use the initial time instant as the initial extreme value time $\tilde{t}^{(0)}=t_{0}$ and use a unit vector as the initial $\operatorname{MPP} \mathbf{u}_{M P P}^{(1)}=\mathbf{u}_{0}$.

Step 2: Perform the MPP search at time instant $\tilde{t}^{(k-1)}$ and obtain MPP $\mathbf{u}_{M P P}^{(k)}$ by solving the following formulas

$$
\begin{cases}\min & \sqrt{\mathbf{U U}^{T}} \\ \text { s.t. } & g\left(T(\mathbf{U}), \tilde{t}^{(k-1)}\right)=0\end{cases}
$$

Step 3: Implement efficient global optimization by fixing $\mathbf{U}$ at $\mathbf{u}_{M P P}^{(k)}$. The optimal time $\tilde{t}^{(k)}$ that minimizes the limit-state function is found and the corresponding minimum value $g_{\min }^{(k)}$ is also obtained.

Step 4: Perform Hessian approximation by using quasiNewton approach with Eq. (24) and envelope theorem with Eq. (28).

Step 5: Check convergence. The convergence criterion is defined as 


$$
\varepsilon=\left|g_{\min }^{(k)}\right| \leq \varepsilon_{t o l}
$$

If $\varepsilon \leq \varepsilon_{\text {tol }}$, terminate the iteration, and $\left(\mathbf{u}_{M P P}^{(k)}, \nabla G^{(k)}, \mathbf{H}^{(k)}\right)$ is the output. Otherwise, set $k=k+1$ and return to step 2 .

Step 6: Calculate $p_{f}$ using SOSPA based on the information $\left(\mathbf{u}_{M P P}^{(k)}, \nabla G^{(k)}, \mathbf{H}^{(k)}\right)$.

The flowchart of overall procedure of SEGO/SOSPA is shown in Fig. 2.

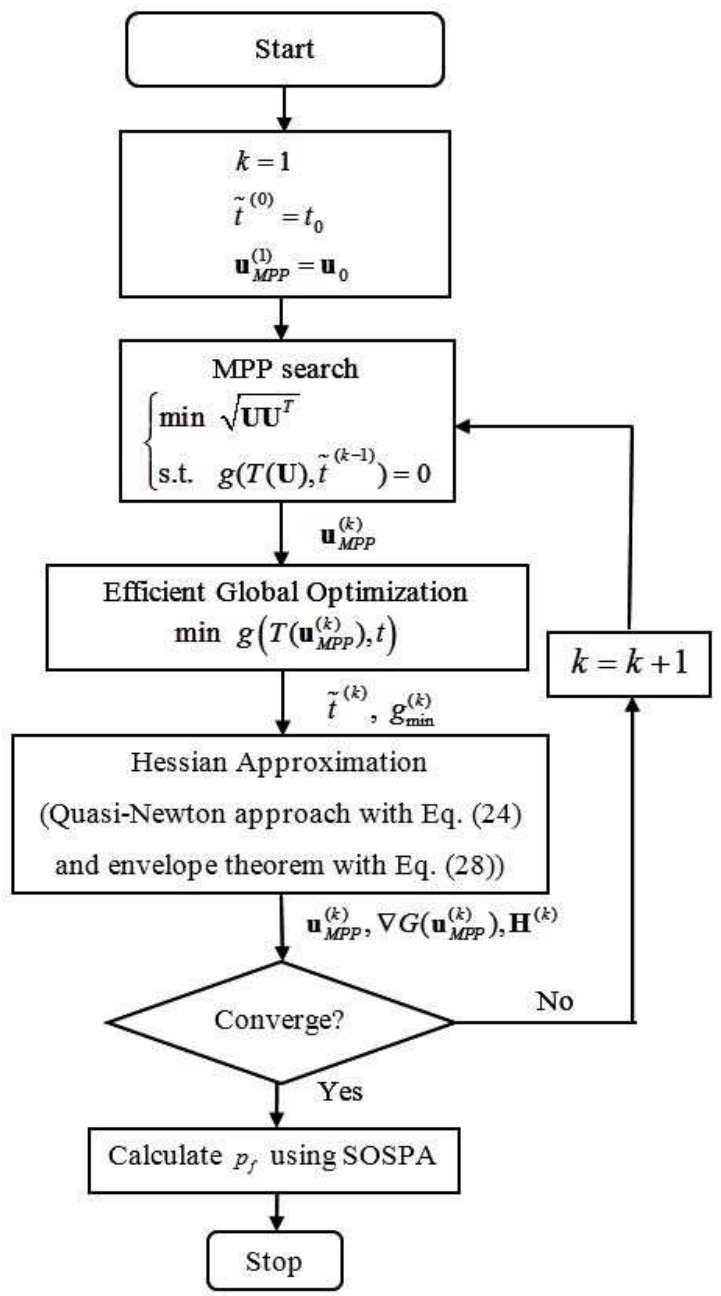

Fig. 2 Flowchart of SEGO/SOSPA

\section{EXAMPLES}

In this section, three examples are used to test SEGO/SOSPA. To show its benefits, we compare it with SEGO using FORM, denoted as SEGO/FORM. The accuracy is evaluated by the relative error with respect to the result from MCS with a large sample size. The relative error is defined as

$$
\varepsilon \%=\left|\frac{p_{f}-p_{f, \mathrm{MCS}}}{p_{f, \mathrm{MCS}}}\right| \times 100 \%
$$

where $p_{f}$ is the result from SEGO/SOSPA or SEGO/FORM. We also use the number of function calls as a measure of efficiency.

\subsection{Example 1: Mathematical Problem}

A mathematical example modified from [27] is used as the first example, which has two independent normal random variables. The limit-state function is given by

$$
g(\mathbf{X}, t)=X_{1}^{2} X_{2}-5 X_{1} t+\left(X_{2}+1\right) t^{2}-9
$$

where $t$ varies within $[0,5], \quad \mathbf{X}=\left[X_{1}, X_{2}\right]$ with $X_{1} \sim N\left(3.5,0.3^{2}\right)$ and $X_{2} \sim N\left(3.5,0.3^{2}\right)$.

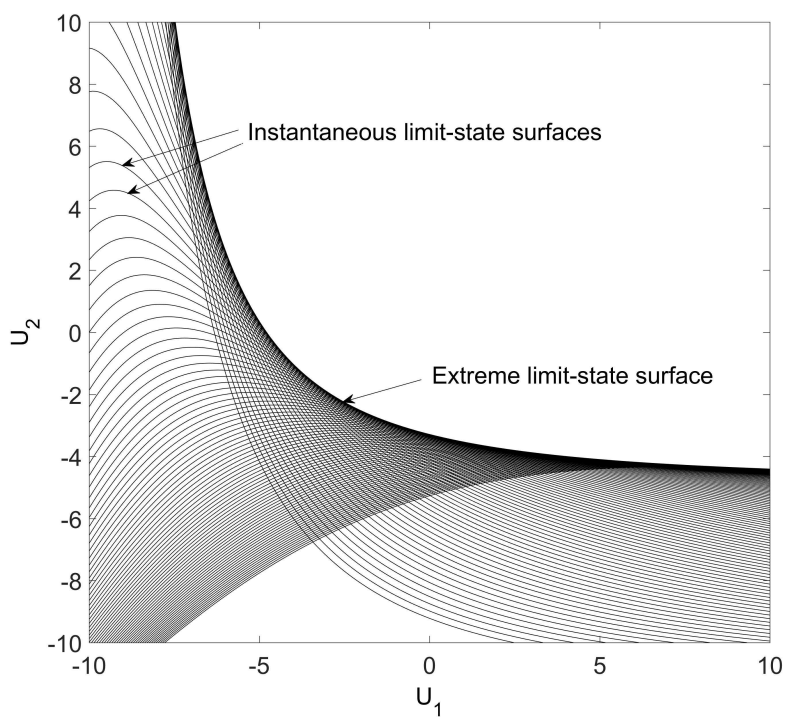

Fig. 3 Extreme limit-state surface formed by instantaneous limit-state surfaces

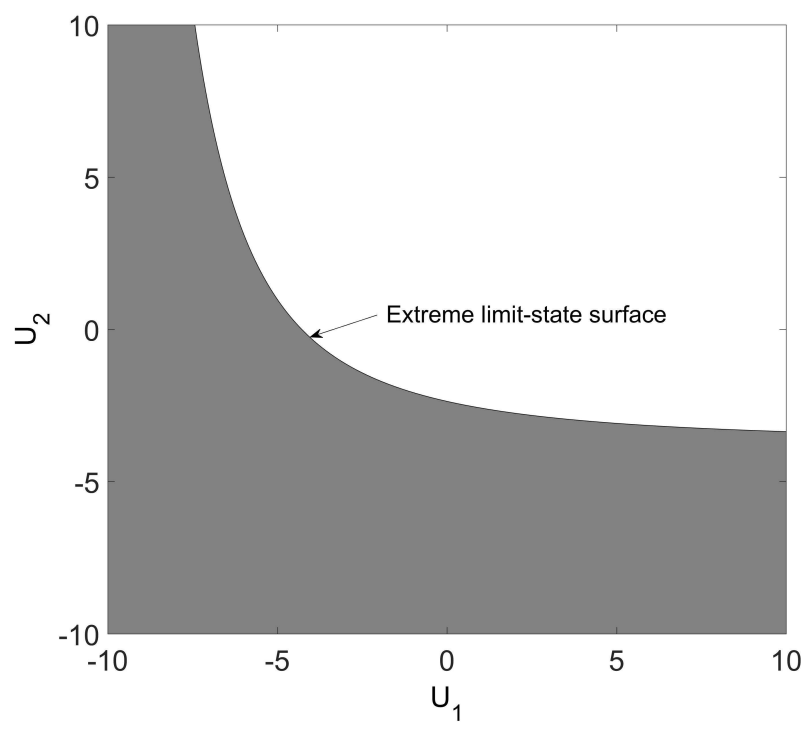

Fig. 4 Extreme limit-state surface 
Fig. 3 shows the extreme failure surface formed by the instantaneous limit-state surfaces at different discretized time instants within the interval $[0,5]$. The extreme limit-state function has a parabolic curve.

The extreme failure surface is confirmed by the one from an analytical equation obtained by solving $\frac{\partial g}{\partial t}=0$, leading to

$$
G(\mathbf{X})=X_{1}^{2} X_{2}-\frac{25 X_{1}^{2}}{4\left(X_{2}-1\right)}-9
$$

The contour of the analytical extreme limit-state function is plotted in Fig. 4, where the failure region is colored grey.

SEGO is used to find the MPP of the extreme limit-sate function. The iteration history of the MPP search is shown in Table 2. Fig. 5 displays the convergence history of first order reliability index $\beta_{F}$ in SEGO. The MPP obtained from SEGO algorithm quickly converges to $(-1.1314,-2.9165)$. It is close to the true MPP at $(-1.1290,-2.9174)$, which is directly obtained from the analytical extreme limit-state function Eq. (42).

Table 2 Iteration history of MPP search for Example 1

\begin{tabular}{lccc}
\hline \hline Iterations & $\mathbf{u}_{M P P}$ & $g_{\min }$ & $\tilde{t}$ \\
\hline 1 & $(-5.8805,-1.7106)$ & -4.1627 & 1.4636 \\
2 & $(-2.1135,-2.7084)$ & -0.8475 & 1.9414 \\
3 & $(-1.4111,-2.8275)$ & -0.0997 & 2.1070 \\
4 & $(-1.2110,-2.8875)$ & -0.0095 & 2.1594 \\
5 & $(-1.1504,-1.1314)$ & $-8.1489 \times 10^{-4}$ & 2.1760 \\
6 & $(-1.1314,-2.9165)$ & $-3.3407 \times 10^{-4}$ & 2.1824 \\
\hline \hline
\end{tabular}

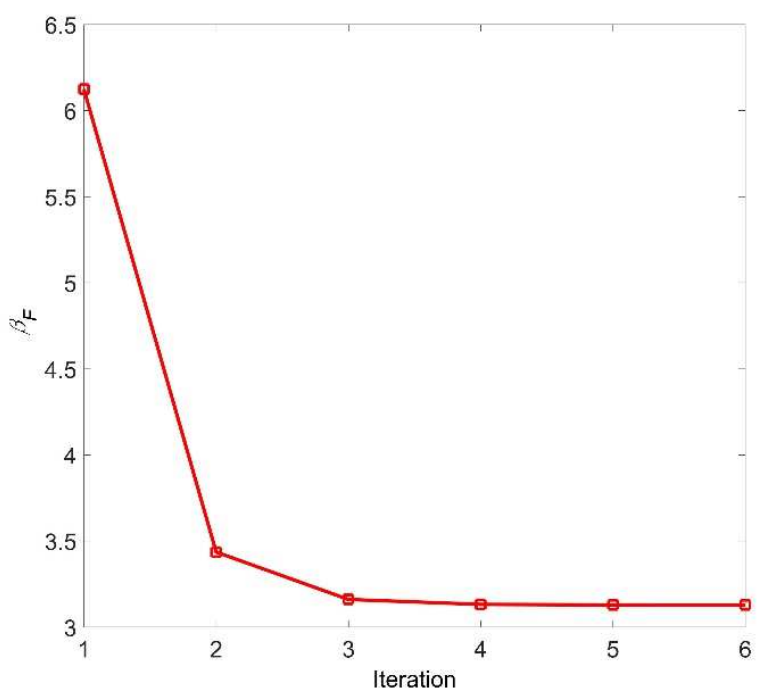

Fig. 5 Convergence history of $\beta_{F}$ in Example 1

After SEGO, FORM and SOSPA are used. For MCS, $10^{6}$ samples are drawn for input random variables $\mathbf{X}$, and the time variable $t$ is discretized evenly into 100 time instants within interval $[0,5]$. The results are shown in Table 3. And they indicate that SEGO/FORM produces a large error of $\varepsilon=18.5 \%$ due to the nonlinearity of the extreme limit-state function. SEGO/SOSPA achieves a more accurate result with an error of $\varepsilon=2.47 \%$. With respect to SEGO/FROM, SEGO/SOSPA requires additional computations which equal to the multiplication of the number of iteration $k$ and the dimension of input random variables $N$, i.e. $k N=6 \times 2=12$. SEGO/SOSPA has much higher accuracy with slightly decreased efficiency.

Table 3 Results of Example 1

\begin{tabular}{lccc}
\hline \hline Methods & $p_{f}$ & $\varepsilon \%$ & $\begin{array}{c}\text { Number of } \\
\text { function calls }\end{array}$ \\
\hline SEGO/SOSPA & $1.0524 \times 10^{-3}$ & $2.47 \%$ & 124 \\
SEGO/FORM & $8.7918 \times 10^{-4}$ & $18.5 \%$ & 112 \\
MCS & $1.0790 \times 10^{-3}$ & - & $10^{8}$ \\
\hline \hline
\end{tabular}

\subsection{Example 2: Automobile Front Axle}

An automobile front axle beam [47] is subjected to a torque $T$ and a bending moment $M=M_{0}(0.1 \sin (0.25 t)+0.9) \mathrm{N} \cdot \mathrm{mm}$ in which $t \in[0,12]$. The limit-state function is given by

$$
g(\mathbf{X}, t)=S_{y}-\sqrt{\sigma^{2}+3 \tau^{2}}=S_{y}-\sqrt{\left(\frac{M}{W_{x}}\right)^{2}+3\left(\frac{T}{W_{\rho}}\right)^{2}}
$$

in which $S_{y}$ is the yield strength, $\sigma$ and $\tau$ are the maximum normal stress and shear stress, respectively, and $W_{x}$ and $W_{\rho}$ are section factor and polar section factor given by

$$
\begin{gathered}
W_{x}=\frac{a(h-2 c)^{3}}{6 h}+\frac{b}{6 h}\left[h^{3}-(h-2 c)^{3}\right] \\
W_{\rho}=0.8 b c^{2}+\frac{0.4 a^{3}(h-2 c)}{c}
\end{gathered}
$$

where $a, b, c$ and $h$ are dimension variables of the I-beam. All the parameters are independent and are listed in Table 4.

Table 4 Distribution of parameters for axle beam example

\begin{tabular}{cccc}
\hline \hline $\begin{array}{c}\text { Variable } \\
(\text { Unit })\end{array}$ & Distribution & Mean & $\begin{array}{c}\text { Standard } \\
\text { Deviation }\end{array}$ \\
\hline$a(\mathrm{~mm})$ & Normal & 12 & 0.6 \\
$b(\mathrm{~mm})$ & Normal & 65 & 3.25 \\
$c(\mathrm{~mm})$ & Normal & 14 & 0.7 \\
$h(\mathrm{~mm})$ & Normal & 85 & 4.25 \\
$M_{0}(\mathrm{~N} \cdot \mathrm{mm})$ & Lognormal & $7 \times 10^{6}$ & $7 \times 10^{5}$ \\
$T(\mathrm{~N} \cdot \mathrm{mm})$ & Extreme value type I & $3.1 \times 10^{6}$ & $3 \times 10^{5}$ \\
$S_{y}(\mathrm{MPa})$ & Deterministic & 610 & - \\
\hline \hline
\end{tabular}

$10^{6}$ samples are used for MCS and $t$ is discretized into 100 time instants within interval $[0,12]$. Results are given in Table 5. Even though SEGO/FORM is more efficient than SEGO /SOSPA, it produces a relatively large error. SEGO/SOSPA is more accurate with only 18 additional function calls compared to $\mathrm{SEGO/FORM.}$ 
Table 5 Results of Example 2

\begin{tabular}{lccc}
\hline \hline Methods & $p_{f}$ & $\varepsilon \%$ & $\begin{array}{c}\text { Number of } \\
\text { function calls }\end{array}$ \\
\hline SEGO/SOSPA & $4.3800 \times 10^{-3}$ & $0.37 \%$ & 176 \\
SEGO/FORM & $4.1899 \times 10^{-3}$ & $4.69 \%$ & 158 \\
MCS & $4.3960 \times 10^{-3}$ & - & $10^{8}$ \\
\hline \hline
\end{tabular}

\subsection{Example 3: A Vibration Problem}

This example involves a forced vibration of a two degree of freedom system modified from $[22,48]$. The original one degree of freedom system is the main system and consists of a spring $k_{1}$ and a mass $m_{1}$. The added system is an absorber and consists of a spring $k_{2}$, a mass $m_{2}$ and a damper $c_{2}$. All parameters are random variables and their distributions are given in Table 6.

Table 6 Distribution of parameters for vibration example

\begin{tabular}{cccc}
\hline \hline $\begin{array}{c}\text { Variable } \\
(\mathrm{Unit})\end{array}$ & Distribution & Mean & $\begin{array}{c}\text { Standard } \\
\text { Deviation }\end{array}$ \\
\hline$k_{1}(\mathrm{~N} / \mathrm{m})$ & Normal & $3 \times 10^{6}$ & $3 \times 10^{5}$ \\
$m_{1}(\mathrm{~kg})$ & Normal & $2 \times 10^{4}$ & $2 \times 10^{2}$ \\
$k_{2}(\mathrm{~N} / \mathrm{m})$ & Normal & $8.5 \times 10^{4}$ & $8 \times 10^{3}$ \\
$m_{2}(\mathrm{~kg})$ & Normal & 480 & 5 \\
$c_{2}(\mathrm{Ns} / \mathrm{m})$ & Normal & 300 & 5 \\
\hline \hline
\end{tabular}

The mass $m_{1}$ in the main system is subjected to a sinusoidal force $f_{0} \sin (\Omega t)$ and the amplitude of vibration is given by

$$
q_{1, \max }=f_{0}\left[\frac{c_{2}^{2} \Omega^{2}+\left(k_{2}-m_{2} \Omega^{2}\right)^{2}}{\left(\begin{array}{l}
c_{2}^{2} \Omega^{2}\left(k_{1}-m_{1} \Omega^{2}-m_{2} \Omega^{2}\right)^{2} \\
+\left(k_{2} m_{2} \Omega^{2}-\left(k_{1}-m_{1} \Omega^{2}\right)\left(k_{2}-m_{2} \Omega^{2}\right)\right)^{2}
\end{array}\right)}\right]^{1 / 2}
$$

Eq. (46) may be non-dimensionalised using a "static" deflection of main system, and the non-dimensional displacement of mass $m_{1}$ is obtained as

$$
\begin{aligned}
\delta & =\frac{q_{1, \max }}{f_{0} / k_{1}} \\
& =k_{1}\left[\frac{c_{2}^{2} \Omega^{2}+\left(k_{2}-m_{2} \Omega^{2}\right)^{2}}{\left(\begin{array}{l}
c_{2}^{2} \Omega^{2}\left(k_{1}-m_{1} \Omega^{2}-m_{2} \Omega^{2}\right)^{2} \\
+\left(k_{2} m_{2} \Omega^{2}-\left(k_{1}-m_{1} \Omega^{2}\right)\left(k_{2}-m_{2} \Omega^{2}\right)\right)^{2}
\end{array}\right)}\right]^{1 / 2}
\end{aligned}
$$

where $\delta$ is the displacement and is considered over a wide excitation frequency band $12 \leq \Omega \leq 30(\mathrm{rad} / \mathrm{s}) . \Omega$ is the excitation frequency and is treated as the time variable $t$ within interval $[12,30] \mathrm{rad} / \mathrm{s}$. A failure occurs when the displacement $\delta$ is larger than 30 . The probability of failure is given by

$$
p_{f}=\operatorname{Pr}\{g(\mathbf{X}, \Omega)=30-\delta<0, \exists \Omega \in[12,30]\}
$$

where $\mathbf{X}=\left[k_{1}, m_{1}, k_{2}, m_{2}, c_{2}\right]$.

SEGO/FORM and SEGO/SOSPA are used to calculate the probability of failure. For MCS, $10^{6}$ samples are used and the time variable $\Omega$ is discretized evenly into 500 instants within the interval $[12,30]$. Table 7 shows the results from different methods. The results indicate that SEGO/SOSPA achieves a higher accuracy than SEGO/FORM while it needs 20 additional function calls.

Table 7 Results of Example 3

\begin{tabular}{lccc}
\hline \hline Methods & $p_{f}$ & $\varepsilon \%$ & $\begin{array}{c}\text { Number of } \\
\text { function calls }\end{array}$ \\
\hline SEGO/SOSPA & $2.5572 \times 10^{-1}$ & $0.06 \%$ & 264 \\
SEGO/FORM & $3.2577 \times 10^{-1}$ & $27.5 \%$ & 244 \\
MCS & $2.5555 \times 10^{-1}$ & - & $5 \times 10^{8}$ \\
\hline \hline
\end{tabular}

\section{CONCLUSIONS}

A new time-dependent reliability method, sequential efficient global optimization/second order saddlepoint approximation, is proposed for limit-state functions which are explicit with respect to time. This new method employs sequential efficient global optimization (SEGO) to convert a time-dependent problem into a time-independent counterpart where the most probable point (MPP) of the extreme limit-state function is obtained. Then a quasi-Newton approach and the envelope theorem are introduced to approximate the Hessian matrix of the extreme limit-state function. Finally the second order saddplepoint approximation (SOSPA) is used to evaluate the probability of failure.

The new method improves the accuracy of time-dependent reliability analysis with a reasonably increased computational effort. It is generally more accurate than the SEGO with first order reliability method (FORM) due to the second-order approximation to the extreme limit-state function. Therefore the new method can be applied to the problems in which extreme limit-state functions are not close to linear. The new method, however, is less efficient than the first order approximation method because it requires second derivatives of extreme limitstate function. But the increase in the computational cost is minimized by the Hessian approximation method and envelope theorem, which make the new method more efficient than the direct second-order approximation.

The proposed method can also be extended to the timedependent problem where the random processes are involved. One feasible solution is to expand and approximate the random processes by sums of random variables. 
Since the proposed method transforms the time-dependent problem into the time-independent one using the extreme value, the accuracy may not be good when multiple local optimums exist. And this method also shares the same limitations of SOSPA. For example, it may not be accurate if the extreme limitstate function is highly nonlinear and far away from a quadratic function.

Our future work includes applying the proposed method into time-dependent reliability-based design and extending the idea to more general limit-state functions.

\section{ACKNOWLEDGEMENTS}

The authors wound like to gratefully acknowledge the support from the National Science Foundation under Grant Number CMMI 1923799.

\section{References}

[1] Choi, S.-K., Grandhi, R. V., and Canfield, R. A., 2006, Reliability-Based Structural Design, Springer Science \& Business Media.

[2] Ditlevsen, O., 2002, "Stochastic Model for Joint Wave and Wind Loads on Offshore Structures," Structural Safety, 24(2), pp. 139-163.

[3] Hime, W., Backus, L., and Li, C., 1999, "Modeling Time-toCorrosion Cracking in Chloride Contaminated Reinforced Concrete Structures. Discussions and Closure," ACI Materials Journal, 96(5).

[4] Suo, Q., and Stewart, M. G., 2009, "Corrosion Cracking Prediction Updating of Deteriorating RC Structures Using Inspection Information," Reliability engineering \& system safety, 94(8), pp. 1340-1348.

[5] Zhang, J., and Du, X., 2011, "Time-Dependent Reliability Analysis for Function Generator Mechanisms," Journal of Mechanical Design, 133(3), p. 031005.

[6] Du, X., 2014, "Time-Dependent Mechanism Reliability Analysis with Envelope Functions and First-Order Approximation," Journal of Mechanical Design, 136(8), p. 081010.

[7] Andrieu-Renaud, C., Sudret, B., and Lemaire, M., 2004, "The PHI2 Method: A Way to Compute Time-Variant Reliability," Reliability Engineering \& System Safety, 84(1), pp. 75-86.

[8] Hu, Z., and Du, X., 2012, "Reliability Analysis for Hydrokinetic Turbine Blades," Renewable Energy, 48, pp. 251262.

[9] Hagen, Ø., and Tvedt, L., 1991, "Vector Process OutCrossing as Parallel System Sensitivity Measure," Journal of engineering mechanics, 117(10), pp. 2201-2220.

[10] Engelund, S., Rackwitz, R., and Lange, C., 1995, "Approximations Of First-Passage Times for Differentiable Processes Based on Higher-Order Threshold Crossings," Probabilistic Engineering Mechanics, 10(1), pp. 53-60.

[11] Streicher, H., and Rackwitz, R., 2004, "Time-Variant Reliability-Oriented Structural Optimization and A Renewal Model for Life-Cycle Costing," Probabilistic Engineering Mechanics, 19(1), pp. 171-183.
[12] Lutes, L. D., and Sarkani, S., 2009, "Reliability Analysis of Systems Subject to First-Passage Failure," NASA Technical Report No. NASA/CR-2009-215782.

[13] Singh, A., Mourelatos, Z. P., and Li, J., 2010, "Design for Lifecycle Cost Using Time-Dependent Reliability," Journal of Mechanical Design, 132(9), p. 091008.

[14] Zhang, J., Wang, J., and Du, X., 2011, "Time-Dependent Probabilistic Synthesis for Function Generator Mechanisms," Mechanism and Machine Theory, 46(9), pp. 1236-1250.

[15] Condra, L., 2001, Reliability Improvement with Design of Experiment, CRC Press.

[16] Holman, J. P., and Gajda, W. J., 2001, Experimental Methods for Engineers, McGraw-Hill New York.

[17] Lundstedt, T., Seifert, E., Abramo, L., Thelin, B., Nyström, Å., Pettersen, J., and Bergman, R., 1998, "Experimental Design and Optimization," Chemometrics and Intelligent Laboratory Systems, 42(1), pp. 3-40.

[18] Papadrakakis, M., and Lagaros, N. D., 2002, "ReliabilityBased Structural Optimization Using Neural Networks and Monte Carlo Simulation," Computer methods in applied mechanics and engineering, 191(32), pp. 3491-3507.

[19] Deng, J., Gu, D., Li, X., and Yue, Z. Q., 2005, "Structural Reliability Analysis for Implicit Performance Functions Using Artificial Neural Network," Structural Safety, 27(1), pp. 25-48.

[20] Hu, Z., Li, H., Du, X., and Chandrashekhara, K., 2013, "Simulation-Based Time-Dependent Reliability Analysis for Composite Hydrokinetic Turbine Blades," Structural and Multidisciplinary Optimization, 47(5), pp. 765-781.

[21] Li, R., and Ghanem, R., 1998, "Adaptive Polynomial Chaos Expansions Applied to Statistics of Extremes in Nonlinear Random Vibration," Probabilistic engineering mechanics, 13(2), pp. 125-136.

[22] Hu, Z., and Du, X., 2015, "Mixed Efficient Global Optimization for Time-Dependent Reliability Analysis," Journal of Mechanical Design, 137(5), p. 051401.

[23] Hu, Z., and Mahadevan, S., 2016, "A Single-Loop Kriging Surrogate Modeling for Time-Dependent Reliability Analysis," Journal of Mechanical Design, 138(6), p. 061406.

[24] Martin, J. D., and Simpson, T. W., 2005, "Use of Kriging Models to Approximate Deterministic Computer Models," AIAA Journal, 43(4), pp. 853-863.

[25] Zhang, J., and Du, X., 2015, "Time-Dependent Reliability Analysis for Function Generation Mechanisms with Random Joint Clearances," Mechanism and Machine Theory, 92, pp. 184199.

[26] Zhu, Z., and Du, X., 2016, "Reliability Analysis with Monte Carlo Simulation and Dependent Kriging Predictions," Journal of Mechanical Design, 138(12), p. 121403.

[27] Wang, Z., and Wang, P., 2012, "A Nested Extreme Response Surface Approach for Time-Dependent ReliabilityBased Design Optimization," Journal of Mechanical Design, 134(12), p. 121007.

[28] Li, J., Chen, J.-b., and Fan, W.-1., 2007, "The Equivalent Extreme-Value Event and Evaluation of The Structural System Reliability," Structural Safety, 29(2), pp. 112-131. 
[29] Singh, A., and Mourelatos, Z. P., 2010, "On the TimeDependent Reliability of Non-Monotonic, Non-Repairable Systems," SAE International Journal of Materials and Manufacturing, 3(2010-01-0696), pp. 425-444.

[30] Hu, Z., and Du, X., 2018, "Saddlepoint Approximation Reliability Method for Quadratic Functions in Normal Variables," Structural Safety, 71, pp. 24-32.

[31] Hu, Z., and Du, X., 2018, "Efficient Reliability-Based Design with Second Order Approximations," Engineering Optimization, pp. 1-19.

[32] Hu, Z., and Du, X., "A Random Field Method for TimeDependent Reliability Analysis With Random and Interval Variables," Proc. ASME 2016 International Design Engineering Technical Conferences and Computers and Information in Engineering Conference, American Society of Mechanical Engineers, pp. V02BT03A044-V002BT003A044.

[33] Du, X., and Chen, W., 2004, "Sequential Optimization and Reliability Assessment Method for Efficient Probabilistic Design," Journal of Mechanical Design, 126(2), pp. 225-233.

[34] Jones, D. R., Schonlau, M., and Welch, W. J., 1998, "Efficient Global Optimization of Expensive Black-Box Functions," Journal of Global optimization, 13(4), pp. 455-492. [35] Lockwood, B., and Mavriplis, D., 2013, "Gradient-Based Methods for Uncertainty Quantification in Hypersonic Flows," Computers \& Fluids, 85, pp. 27-38.

[36] Raghavan, B., and Breitkopf, P., 2013, "Asynchronous Evolutionary Shape Optimization Based on High-Quality Surrogates: Application to An Air-Conditioning Duct," Engineering with Computers, 29(4), pp. 467-476.

[37] Arora, J. S., 2004, Introduction to optimum design, Elsevier. [38] Lim, J., Lee, B., and Lee, I., 2014, "Second-Order Reliability Method-Based Inverse Reliability Analysis Using Hessian Update for Accurate and Efficient Reliability-Based Design Optimization," International Journal for Numerical Methods in Engineering, 100(10), pp. 773-792.
[39] Chambers, R. G., Chung, Y., and Färe, R., 1996, "Benefit and Distance Functions," Journal of economic theory, 70(2), pp. 407-419.

[40] Carter, M., 2001, Foundations of mathematical economics, MIT Press.

[41] Breitung, K., 1984, "Asymptotic Approximations for Multinormal Integrals," Journal of Engineering Mechanics, 110(3), pp. 357-366.

[42] Tvedt, L., 1990, "Distribution of Quadratic Forms in Normal Space - Application to Structural Reliability," Journal of Engineering Mechanics, 116(6), pp. 1183-1197.

[43] Daniels, H. E., 1987, "Tail Probability Approximations," International Statistical Review/Revue Internationale de Statistique, pp. 37-48.

[44] Goutis, C., and Casella, G., 1999, "Explaining the Saddlepoint Approximation," The American Statistician, 53(3), pp. 216-224.

[45] Du, X., and Sudjianto, A., "A Saddlepoint Approximation Method for Uncertainty Analysis," Proc. ASME 2004 International Design Engineering Technical Conferences and Computers and Information in Engineering Conference, American Society of Mechanical Engineers, pp. 445-452.

[46] Hu, Z., and Du, X., 2019, "Reliability Methods for Bimodal Distribution with First-Order Approximation," ASCE-ASME Journal of Risk and Uncertainty in Engineering Systems, Part B: Mechanical Engineering, 5(1), p. 011005.

[47] Shi, Y., Lu, Z., Cheng, K., and Zhou, Y., 2017, "Temporal and Spatial Multi-Parameter Dynamic Reliability and Global Reliability Sensitivity Analysis Based on The Extreme Value Moments," Structural and Multidisciplinary Optimization, 56(1), pp. 117-129.

[48] Zang, C., Friswell, M., and Mottershead, J., 2005, "A Review of Robust Optimal Design and Its Application in Dynamics," Computers \& structures, 83(4-5), pp. 315-326. 